\title{
The influence of mating system on the intensity of parent-offspring conflict in primates
}

\author{
T. A. F. LONG \\ Department of Biology, Queen's University, Kingston, Ontario, Canada
}

\author{
Keywords: \\ foetal growth rate; \\ mating system; \\ parent-offspring conflict; \\ parental investment; \\ placental structure; \\ primates; \\ sperm competition.
}

\begin{abstract}
An evolutionary conflict of interest exists between parents and their offspring over the partitioning of parental investment (PI) among siblings. When the direct fitness benefits to offspring of increased PI, outweigh the inclusive fitness costs from lost future sibling fitness, selection should favour the evolution of offspring selfishness over altruism. In theory, this conflict is heightened when females are not strictly monogamous, as current offspring should be less altruistic towards future half-siblings than they would be towards full-siblings. Using data collected on foetal growth rate (representing prenatal PI) in primates, I test the prediction from theory that the resolution of the parent-offspring conflict will be closer to the offspring's evolutionary optima in polyandrous species than in more monandrous species. Using phylogenetic comparative analysis, and controlling for allometry, I show that offspring are able to obtain more PI when the probability of future full-siblings decreases, and that this is most pronounced in taxa where there is the opportunity for direct foetal access to the maternal bloodstream. These results support the hypothesis that the resolution of prenatal PI conflict is influenced by both a species' mating system and by its placental structure.
\end{abstract}

\section{Introduction}

Thirty years ago, Trivers (1974) overturned the classic evolutionary framework although which biologists viewed the relationship between parents and their offspring. Instead of being purely co-operative in nature, he showed that there was considerable potential for discord between the two parties over the amount of parental investment (PI) appropriated, once offspring were viewed as active participants in the consociation, rather than as passive vessels into which parental resources are poured. The key to understanding the dynamics of this relationship lay in the realization that in a sexually reproducing diploid species a mother and her child are not genetically identical, but instead have a coefficient of relatedness $(r)$ of exactly 0.5 . By applying Hamilton's (1964) rule, developed to explain the evolution of altruism via kin selection, Trivers (1974) described

Correspondence: T. A. F. Long, Department of Biology, Queen's University, Kingston, Ontario, Canada, K7L 3N6.

Tel.: (613) 533-6000 ext. 75134; fax: (613) 533-6617;

e-mail: longt@biology.queensu.ca the conditions necessary for the spread of 'selfish' genes that benefit the offspring through increased PI, with a cost to the parents' lifetime reproductive success.

In theory, natural selection will favour selfishness in offspring up until the point where the indirect fitness costs outweigh the direct benefits of increased PI (Trivers, 1974). The upper limit of this selfishness is defined by the value of the coefficient of relatedness between the current offspring and its future siblings. For instance, when all future siblings will be fathered by the same individual (i.e. will be full-siblings), the indirect costs to the current offspring are discounted by a factor equal to the average coefficient of relatedness (i.e. $r=0.5$ ). In species where females are not strictly monogamous, and instead mate with multiple, unrelated males, there is a much greater likelihood that, on average, future offspring will be half-siblings, $(r=0.25)$. In such a species, the spread of selfish genes should be less constrained than in a monogamous species, as current offspring share a smaller fraction of the indirect fitness costs of selfishness, due to their lower coefficient of relatedness with their future siblings (Trivers, 1974). In both situations, the mother will be equally related 


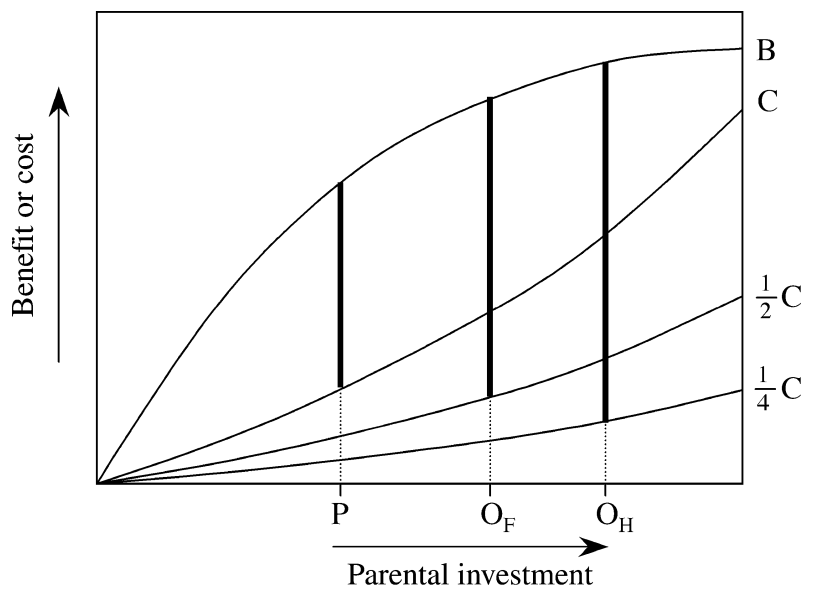

Fig. 1 The benefit (B), cost $(\mathrm{C})$, half the cost $(1 / 2 \mathrm{C})$ and a quarter the cost $(1 / 4 \mathrm{C})$ of a parental act towards and offspring as a function of the amount of investment in the act. At the point $\mathrm{P}$, the parent's inclusive fitness is maximized $(\mathrm{B}-\mathrm{C})$. At the point $O_{\mathrm{F}}$ the offspring's inclusive fitness is maximized if all future offspring are full-siblings $\left(r_{\mathrm{o}}=0.5\right)$. At the point $O_{\mathrm{H}}$ the offspring's inclusive fitness is maximized if all future offspring are half-siblings $(r=0.25)$. Since $O_{\mathrm{H}}>O_{\mathrm{F}}$, there is a larger potential zone of conflict between the parent's optimal investment level and the offspring's optima in species where there is polyandry than in species with monogamy (Godfray, 1995; modified from Trivers, 1974).

$(r=0.5)$ to all of her offspring, but there will be a greater disparity between the parent and the offspring's interests in the promiscuous species than in the monogamous species (Fig. 1).

From the moment of conception to the moment of birth, the foetus is entirely dependent on its mother, with the placenta acting as a conduit, to provide the resources necessary for growth and development and removal of waster products (see Harding \& Johnston, 1995 for review). The size of the investment made early in life by the parent is of central importance to the lifetime fitness of the offspring (Widdowson, 1974; Lindström, 1999). Offspring that receive less provisioning during the gestation period tend to be smaller at birth (Harding $\delta$ Johnston, 1995), stay small throughout life (Widdowson, 1974), and have an increased risk of adult disease (e.g. Leon et al., 1998; Hyppönen et al., 2001) and or mortality (e.g. Geissmann \& Orgeldinger, 1995). A recent review (e.g. Lummaa, 2003) of these 'downstream effects' concluded that even slight differences in neonatal mass might have important fitness repercussions: larger babies tend to grow up to be fitter adults. Thus, it may be in the offspring's selfish interests to attempt to increase its resource acquisition during the gestation period, while, for the reasons described above the parent may be selected for more restraint.

Given the differences in evolutionary optima between a mother and her offspring over the allocation of resources, Parker \& Macnair (1979) investigated how such a conflict can be resolved using an evolutionary stable strategy (ESS) modelling approach. In their model, both parties are able to respond to the strategy played by the other. They envisioned an ESS solution wherein parents adopt a pro rata strategy: providing only a proportion of the offspring's demands that exceeds their own optimal PI, but is not as extensive as the offspring PI requests. Thus, a compromise on the 'battleground' is reached between the two parties: in exchange for reducing the relative amount of solicitation by the offspring, the parent responds by making more resources available (Parker \& Macnair, 1979; Godfray, 1995; Mock \& Parker, 1997). Parker \& Macnair (1979) compared the pro rata ESS levels of PI for a species that was monogamous, with one that had female promiscuity, and found that the quantity of PI delivered at the point of resolution was much larger in the latter mating system than in the former.

I set out to test empirically the explicit prediction of Parker \& Macnair (1979), that the pro rata resolution of the parent-offspring conflict (POC) will evolve in response to the likelihood of female promiscuity, and the observed amount of PI that is allocated to the offspring will be greater in more promiscuous species than in more monogamous species. This prediction was tested using the comparative method using data collected from species in the Order Primates, as this relatively wellstudied group exhibits considerable variation in life history patterns (Harvey et al., 1987).

\section{Methods}

\section{Currency of conflict: the extent of in utero parental investment}

In this study, the rate of growth of the foetus over the course of the gestation period was used to measure the resolution of conflict between the mother and offspring a compromise between the two parties' evolutionary optima over the amount of resources to be delivered before parturition. Thus, foetal growth rate [neonatal mass $(\mathrm{g}) \div$ gestation length (days)] was used as the index of prenatal PI in all analyses. Although the size if the PI made during gestation period often smaller than the investment made during after birth (Lee, 1996), it still costly to the mother (see Robbins, 1993), and should therefore be subject to the influence of natural selection.

\section{Predicting the likelihood of future full- vs. half- siblings}

I used two different methods to estimate the likelihood that sequential siblings will be fathered by different males based on (1) potential social opportunities for female promiscuity and (2) male morphological adaptations associated with females mating with multiple males.

The first estimate is founded upon the premise that the structure of species' mating system provides, on average, 
different opportunities for females to mate with multiple males, based on the presence or absence of multiple adult males in the social group during the breeding season. Thus, species were categorized as having either only one male (harem group and monogamous mating systems) or more than one (multi-male/female and polyandrous mating systems). When conflicting reports of social structure exist (e.g. species where both harem and multi-male/female groups have been reported), the species was assigned to the 'more than one male' group. According to POC theory (Macnair \& Parker, 1978), there is should be no difference from the offspring's perspective between harem group and monogamous systems, as they both are likely to have only one potential father for future offspring. In reality, Primate harems may potentially undergo resident male turnover between breeding episodes, reducing the average relatedness of a female's offspring. Despite the absence of long-term studies for many harem species, it does appear that in some species that the length of resident males is long enough to span multiple breeding events (e.g. Steenbeek, 2000). Thus, the two categories (one male or more than one male) used in this index reflect different relative probabilities of the production of full-siblings, rather than absolute reflections of sibling relatedness.

Due to the limitations inherent in the first index, I also derived an second estimate used to predict the likelihood of half-siblings in a taxa is based on the species' risk of experiencing sperm competition; postcopulatory competition between the ejaculates of rival males for the fertilization of a female's ova (Parker, 1970). Sperm competition risk increases as females mate with more males during a breeding episode (Parker, 1998), and should consequently be inversely related to the likelihood having the same father sire all of a female's offspring. Sperm competition theory (Parker, 1998) predicts that when females mate multiply, with temporal overlap of ejaculates in the female's reproductive tract, males will be selected to evolve traits that improve their chances of increasing their paternity. Often, this selection is manifested as increased investment into the testes (Hosken $\&$ Ward, 2001), and more polyandrous species tend to contain males with relatively larger testes than more monogamous species (e.g. Harcourt et al., 1995).

\section{Data}

Data were collected from a variety of primary and secondary literature sources (e.g. Geissmann \& Orgeldinger, 1995; Harcourt et al., 1995; Lindenfors, 2002; Ernest, 2003; Schülke et al., 2004), as well as from personal communications with primate researchers (see Acknowledgments). I was able to collect data on male and female adult body mass $(n=183)$, foetal growth rate $(n=118)$, mating system $(n=168)$ and combined testes mass $(n=$ $79)$. Where necessary, data on single testis masses were doubled, and values reported as volumes were converted to mass by multiplying by the specific gravity of testes (1.087 $\mathrm{g} \mathrm{cm}^{-3}$; Møller, 1991) so all values would be in the same currency. For analyses based on morphometric data, each testis was assumed to be an ellipsoid (Møller, 1991; Møller \& Briskie, 1995).

All species were coded for whether they possessed either a hemochorial or an epitheliochorial placenta, a categorization that corresponds to the two Primate clades of Haplorhini and Strepsirhini (Luckett, 1975). The hemochorial placenta is characterized by a very intimate association between the foetus and mother: a loss of maternal epithelial and connective tissue from the structure permits direct foetal access to the maternal bloodstream (Luckett, 1975). The ancestral epitheliochorial placenta, on the other hand, is more diffuse, and there are more barriers between the female and foetus (Luckett, 1975). If the fundamental differences in the placental structure affects the nature of the POC (Crespi $\&$ Semeniuk, 2004) in the two clades or affects their lifehistory allometries, it may be inappropriate to consider Primates as a whole. Consequently, all analyses were repeated separately for the Haplorhines and for the Strepsirhines.

\section{Analysis}

Since species that are descended from a common ancestor are likely to possess similar characteristics as a result of 'phylogenetic inertia' (Harvey \& Pagel, 1991), their data should not be treated as independent data points (Felsenstein, 1985). Consequently, I used the CAIC software package (v. 2.6.9; Purvis \& Rambault, 1995), which generated phylogenetically independent data points, using a modified version of Felsenstein's (1985) method, to control for the potentially confounding effects of shared ancestry. I used a recently derived, well-resolved supertree phylogeny of the primates (Vos \& Moores, 2005), and assumed equal branch lengths. Analyses were performed according to the guidelines of the CAIC manual (Purvis \& Rambault, 1995), and where necessary, values were removed if their Studentized residuals were $>1.96 \mathrm{SD}$, in order to maintain homoscedasticity of contrasts (see Jones \& Purvis, 1997). All data were $\log _{10}$-transformed prior to CAIC analysis in order to homogenize variances.

Since a species' life history and its morphological traits are often influenced by its body size (e.g. Leutenegger, 1973; Martin, 1984; Harvey \& CluttonBrock, 1985; Martin \& MacLaron, 1985), it was also necessary to control for the potentially confounding effects of allometry on the data. Following the guidelines of the CAIC manual (Purvis \& Rambault, 1995), when the ultimate predictor was categorical, I first derived the phylogentically controlled relationship between maternal mass and foetal growth rate using the CRUNCH algorithm, derived the regression 
equation, and then used this formula to calculate residual values from the raw, uncontrolled data. These residual values were then tested against the categorical variable, using the BRUNCH algorithm. For the relationship between foetal growth rate (FGR) and risk of sperm competition, all four variables (FGR, testes mass, male mass, female mass) were analysed in CAIC using CRUNCH, and then the partial correlation between foetal growth rate and testes mass, (partialled with respect to both male and female mass) was calculated.

To test whether the direction of the phylogenetic contrasts was significant after BRUNCH analysis, values were randomly sampled 1000 times (Blank, 2001) and tested to see if evolution had followed random Brownian motion $\left(H_{0}\right.$ : mean contrast $\left.=0\right)$ or was directional in nature $\left(H_{1}\right.$ : mean contrast $\neq 0$ ) (Harvey \& Pagel, 1991).

\section{Results}

\section{Does foetal growth rate differ between species with social availability of adult males?}

After controlling for phylogenetic and allometric relationships and removing one outlier, there were 16 contrasts between taxa with differing social numbers of males. Species where polyandry was possible had a greater prenatal growth rates than species where only one father was presumed [mean contrast $\log (\mathrm{FGR})=$ $0.032 ; 95 \% \mathrm{CI}=0.024-0.039]$. Without any phylogenetic controls, there was also a significant difference between the monadrous [mean residual $\log (\mathrm{FGR})=$ -0.07] and polyandrous groups [mean residual $\log (\mathrm{FGR})=0.03](t=3.322$, d.f. $=111, P<0.01)$.

Due to the phylogenetic distribution of the structures, it is not possible to use CAIC to compare foetal growth rate between the two placental types as only one contrast is possible. If no phyogenetic correction is applied, and female-mass-corrected $\log (\mathrm{FGR})$ values are compared, the hemochorial Haplorhines [mean $\log (\mathrm{FGR})=0.051$ ] have greater foetal growth rates than the epitheliochorical Strepsirhines [mean $\log (\mathrm{FGR})=-0.139](t=6.104$, d.f. $=116, P<0.001)$.

When the Haplorhines and Strepsirhines are considered separately, the same difference in relative FGR seen at the order level was also seen in the Haplorhines (12 contrasts, one outlier removed), with the species with multiple adult males growing faster than those with only one adult male [mean difference in relative $\log (\mathrm{FGR})$ contrasts $=0.039, \quad t=20.924, \quad$ d.f. $=11, \quad P<0.001]$. Without any phylogenetic controls, there was also a significant difference between the monadrous and polyandrous Haplorhines groups $(t=1.89$, d.f. $=80$, $P<0.05)$. In the Strepsirhines, however, only four contrasts were generated, and no difference between FGR monandrous and polyandrous groups was detected [mean difference in relative $\log (\mathrm{FGR})$ constrasts $=$ $-0.180, t=0.661$ d.f. $=3$, n.s.]. Without any phylo- genetic controls, there was still no difference in the residual $\log (\mathrm{FGR})$ means between the two groups in the Strepsirhines $(t=0.09$, d.f. $=29$, n.s. $)$. In order to test for potential confounds that may be due to sexual dimorphism, the female-mass corrected FGR values were regressed on $\log$ (male mass) values using the CRUNCH algorithm. No significant positive correlation between residual FGR and male mass was detected in either the Haplorhine group $(r=-0.06$, d.f. $=81$ n.s.; without phylogenetic control $r=-0.187$, d.f. $=87, P=0.08$ ) or in the Strepsirhine group $(r=-0.24$, d.f. $=22$, n.s.; without phylogenetic control $r=-0.22$, d.f. $=24$, n.s.), indicating that the variation in FGR cannot be attributed to sexual dimorphism.

\section{Does foetal growth rate vary with risk of sperm competition?}

When all primates were analysed together, distributions were non-normal (Shapiro-Wilks tests, $P<0.05$ ), so nonparametric correlations were calculated. All four continuous variables co-varied with each other (Table 1). Foetal growth rate and testes mass positively correlated with each other, but this relationship does not take either the correlated effects of male or female body mass into account. The second-order Kendall partial Tau b correlation (SAS Institute Inc., 1999) for $\log (\mathrm{FGR})$ and $\log$ (testes mass) with the adult masses of the two sexes taken into account was 0.066 , which was not significant (one-tailed test; d.f. $=63$; Tau critical $_{\alpha=0.05}=0.12 ;$ n.s.).

When the analyses were performed separately on the Haplorhines and Strepsirhines, different patterns became apparent. Both groups could be analysed using parametric methods. One set of contrasts (between the Tarsioidea and the rest of the Haplorhini group) was removed from the Haplorhine group as it was identified as an extreme outlier (Mahalanobis distance $>6.5$ ). In the Haplorhine group ( $n=40$ contrasts), there was a significant positive correlation between foetal growth rate and combined testes mass that was independent of adult male and female mass (Table 2, Fig. 2). The inclusion of the outlying contrast did not dramatically affect this correlation (Pearson partial correlation $=0.27$, d.f. $=37$, $P<0.05)$. In the Strepsirhine group $(n=25$ contrasts;

Table 1 Correlations between phylogenetically controlled variables for all Primate contrasts $(n=67)$, Coefficient values are Kendall Tau b nonparametric correlations.

\begin{tabular}{lllll}
\hline & Log (female) & Log (male) & $\log (F G R)$ & $\log$ (testes) \\
\hline $\log ($ female) & - & $0.794^{\star \star \star}$ & $0.542^{\star \star \star}$ & $0.263^{\star \star}$ \\
$\log ($ male) & & - & $0.479^{\star \star \star}$ & $0.304^{\star \star \star}$ \\
$\log (F G R)$ & & - & $0.208^{\star}$ \\
$\log$ (testes) & & & - \\
\hline
\end{tabular}

One-tailed tests on correlation coefficients; ${ }^{*} P<0.05 ; * * P<0.005$; $* * * P<0.001$. 
Table 2 Correlations between phylogenetically controlled variables for the Haplorhine contrasts $(n=40)$, Coefficient values above the diagonal are Pearson full correlations and values below the diagonal are partial correlations.

\begin{tabular}{llcll}
\hline & Log (female) & Log (male) & Log (FGR) & Log (testes) \\
\hline Log (female) & - & $0.924^{\star \star}$ & $0.761^{\star \star}$ & $0.500^{\star \star}$ \\
Log (male) & $0.868^{\star \star}$ & - & $0.649^{\star \star}$ & $0.502^{\star \star}$ \\
Log (FGR) & $0.550^{\star \star}$ & -0.259 & - & $0.520^{\star \star}$ \\
Log (testes) & -0.067 & 0.184 & $0.283^{\star}$ & - \\
\hline
\end{tabular}

One-tailed tests on correlation coefficients; ${ }^{*} P<0.05 ; * * P<0.001$.

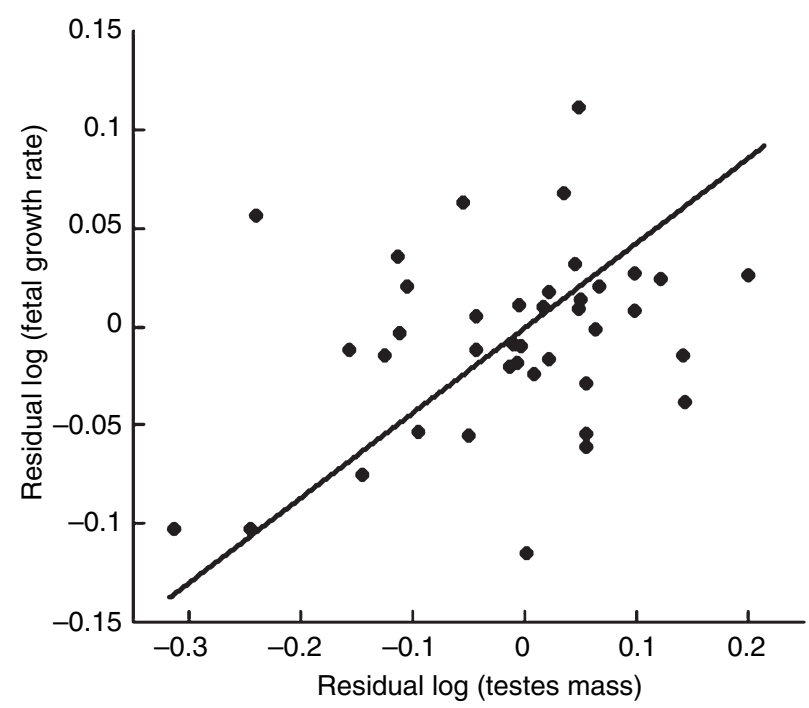

Fig. 2 Independent contrast analysis of the size of prenatal parental investment (relative foetal growth rate) in relation to the likelihood that future offspring will be half-siblings (relative combined testis size). Circles represent phylogentically independent contrasts, corrected for adult body mass, for primates in the Haplorhine group $(n=40$, Pearson partial correlation $=0.283, P<0.005)$. Reduced major axis regression line is shown.

Table 3 Correlations between phylogenetically controlled variables for the Strepsirhine contrasts $(n=25)$, Coefficient values above the diagonal are Pearson full correlations and values below the diagonal are partial correlations.

\begin{tabular}{lllll}
\hline & Log (female) & Log (male) & Log (FGR) & Log (testes) \\
\hline Log (female) & - & $0.983^{\star}$ & $0.665^{\star}$ & 0.246 \\
Log (male) & $0.967^{\star}$ & - & $0.662^{\star}$ & 0.276 \\
Log (FGR) & 0.092 & 0.080 & - & 0.084 \\
Log (testes) & -0.127 & 0.197 & -0.124 & - \\
\hline
\end{tabular}

One-tailed tests on correlation coefficients; ${ }^{*} P<0.001$.

Table 3), however, the partial correlation between FGR and testes mass was much weaker than in the Haplorhines and not statistically significant.

\section{Discussion}

Using a large, comparative data set, I set out to test the hypothesis that the maternal-foetal relationship has been shaped by natural selection in a manner consistent with POC theory. The results, described above, support this explanation. In general, when all Primates were considered as a whole, the features of a species' mating system (presence of rivals in social group and morphological evidence of sperm competition) were somewhat equivocal predictors of variation in PI (as measured by relative foetal growth rate), when potentially confounding allometric and phylogenetic factors were controlled. However, when analyses were restricted to the Haplorhines, differences in prenatal PI were correlated with the probability of relatedness to future offspring. Species with more potential female promiscuity were characterized by a higher rate of foetal growth than in more monogamous species using both indices. Why might we expect to see a positive relationship between female promiscuity in the mating system and relative foetal growth that prominent in the Haplorhines, but is absent in the Strepsirhines? There are a number of potential differences in the life histories ecologies and morphologies of these groups (e.g. Luckett, 1975; Harvey \& Clutton-Brock, 1985). A likely explanation based on evolutionary motive and opportunity reveals a fascinating perspective of how POC battles may be 'fought'.

The escalation and ultimate resolution of the POC may be thought of as having two equally important components: motive and opportunity. The foetus' motive, in the evolutionary sense, refers to the value of pursuing selfish vs. altruistic behaviour over the amount of PI that is requested. This motive is linked to the probability of having full- or half-siblings, which is associated with the likelihood of female promiscuity. Opportunity, in this case, refers to the physical capacity of an offspring to attempt to coerce a greater-than-optimal amount of investment from the parent. Trivers (1974) recognized that there are many situations in which offspring may be unable to manipulate PI allocation, when he wrote that 'an offspring can not fling its mother to the ground at will and nurse' (p. 257). Strepsirhine mothers may, by virtue of their epitheliochorical placental structure be better able to resist biochemical manipulation by the developing foetus. While the hemochorial placenta is characterized by its invasiveness of the uterine vasculature, which permits the foetus access to it mother's nutrients and endocrine system (Haig, 1993), the additional layers of maternal tissue (Luckett, 1975) allow the female to have more protection from potential biochemical manipulation. Even if there is ample evolutionary motive for the evolution of selfish behaviours, the lack of opportunity may constrain the coevolution of the two parties.

Haig (1993) suggested that the structure of the hemochorial placenta was suggestive of an escalation of in utero POC. The results of this study are supportive of this 
conclusion, as the amount of prenatal PI varied with probability of female promiscuity in a manner consistent with theory, in the species possessing the hemochorial placenta. Leutenegger (1973) had suggested that the type of placenta had a role in determining the amount of investment made by the parent in its offspring. However, he (and subsequently Martin, 1984) focused on mass at birth, rather than on relative foetal growth rate, as I have done, and did not take gestation length into account, which may explain the inconsistency of results in interorder comparisons (Leutenegger, 1973; Martin, 1984). Similar results to those described above should be found in other hemochorial groups (such as Orders Rodentia, Lagomorphia, Insectivora and some Chiroptera), while no correlation will be found between relative foetal growth rate and mating system will be found in groups with the less maternally invasive placenta (Orders Carnivora, Artilodactyla, Perissodactyla and Cetacea).

In a study of antagonistic selection on primate size, Lindenfors (2002) included a test that compared foetal growth rates across species with different mating systems and found no differences at either the Order level, or within the Haplorhines separately. There are two possible explanations for having obtained different results. The first may be a matter of statistical power. I was able to generate slightly more phylogenetically independent contrasts for the primates (16 vs. 15) and for the Haplorhines (12 vs. 11) than Lindenfors (2002), in part due to the use of a larger, and more updated, data set. Second, Lindenfors (2002) made a distinction between monogamous and harem group mating systems, which is not necessary in a POC study (Macnair \& Parker, 1978), as both types may be predisposed to producing fullsiblings. It should be noted that Lindenfors' (2002) nonsignificant comparisons of foetal growth rates were in the same direction (faster growth as mating system departs from monogamy) as repeated here.

One pathway by which the offspring's evolutionary interests are thought to be expressed is through the action of parentally imprinted genes, where a gene 'knows' which parent it came from (Haig \& Westoby, 1989; Haig, 1992). Foetal-expressed genes promoting growth (genes whose actions demands greater PI), are thought to be expressed when inherited from the father, and turned off when inherited from the mother, but this will depend on the mating system (Haig, 1992). With female promiscuity, fathers will benefit from increased PI by the mother, as it will mostly come at the expense of unrelated, future, offspring (Trivers, 1974). It thus follows that promiscuous species should have a greater number or activity of imprinted genes than monogamous species (Haig, 1992), a prediction that has gained some experimental support (Vrana et al., 1998).

While the evolutionary arms race between parents and their offspring is well established in theory (Godfray, 1995; Mock \& Parker, 1997), the bulk of empirical evidence for prenatal conflict has come from the study of genomic imprinting. Here I show the potential for tests of POC theory using data from the prenatal period, using the comparative method. Contrary to the suggestion of Harvey et al. (1987), I was able to demonstrate that deviations in neonatal size and gestation length in the primates do have an adaptive explanation, one that is rooted in conflict theory. This study presents another step towards the 'elusive goal' (Harvey et al., 1987) of an overall explanation of primate life history patterns and our understanding of the evolutionary dynamics of the maternal-foetal relationship.

\section{Acknowledgments}

Data on primates on was generously provided by M. Anderson, S. Gursky, S. Meker, L. Nash, W. Olupot, S. Pochron and O. Schülke. R.A. Vos and A.Ø. Moores constructed the primate phylogeny. A. Schülte-Hostedde and A. Purvis provided suggestions on data analysis. A. Chippindale, R. Montgomerie, T. Day, A. Wilczek and the Queen's Behavioral Ecology and Evolution Research Seminar (BEERS) group are thanked for helpful suggestions. TAFL was supported by a NSERC PGS-B graduate scholarship.

\section{References}

Blank, S., Seiter, C. \& Bruce, P. 2001. Resampling Statistics for Excel v. 2.0 [WWW document]. URL http://Www.resample. $\mathrm{com} /$ content/software/excel/index.shtml [accessed on 16 February 2005].

Crespi, B.J. \& Semeniuk, C. 2004. Parent-offspring conflict in the evolution of vertebrate reproductive mode. Am. Nat. 163: 635-653.

Ernest, S.K.M. 2003. Life history characteristics of non-volant placental mammals. Ecology 84: 3401.

Felsenstein, J. 1985. Phylogenies and the comparative method. Am. Nat. 125: 1-15.

Geissmann, T. \& Orgeldinger, M. 1995. Neonatal weight in gibbons (Hylobates spp.). Amer. J. Primat. 36: 179-189.

Godfray, H.C.J. 1995. Evolutionary theory of parent-offspring conflict. Nature 376: 133-138.

Haig, D. 1992. Genomic imprinting and the theory of parentoffspring conflict. Sem. Dev. Biol. 3: 153-160.

Haig, D. 1993. Genetic conflicts in human pregnancy. Q. Rev. Biol. 68: 495-532.

Haig, D. \& Westoby, M. 1989. Parent-specific gene expression and the triploid endosperm. Am. Nat. 134: 147-155.

Hamilton, W.D. 1964. The genetical theory of natural selection. J. Theor. Biol. 7: 1-52.

Harcourt, A.H., Purvis, A. \& Liles, L. 1995. Sperm competition: mating system, not breeding season, affects testes size in Primates. Funct. Ecol. 9: 468-476.

Harding, J.E. \& Johnston, B.M. 1995. Nutrition and fetal growth. Reprod. Fertil. Dev. 7: 539-547.

Harvey, P.H. \& Clutton-Brock, T.H. 1985. Life history variation in Primates. Evology 39: 559-581.

Harvey, P.H. \& Pagel, M.D. 1991. The Comparative Method in Evolutionary Biology. Oxford University Press, Oxford. 
Harvey, P.H., Martin, R.D. \& Clutton-Brock, T.H. 1987. Life histories in comparative perspective. In: Primate Societies (B. B. Smuts, D. L. Cheney, R. M. Seyfarth, R. W. Wrangham \& T. T. Struhsaker, eds), pp. 181-196. University of Chicago Press, Chicago, IL.

Hosken, D.J. \& Ward, P.I. 2001. Experimental evidence for testis size evolution via sperm competition. Ecol. Lett. 4: 10-13.

Hyppönen, E., Leon, D.A., Kenward, M.G. \& Lithell, H. 2001. Prenatal growth and risk of occlusive and haemorrhagic stroke in Swedish men and women born 1915-29: historical cohort study. BMJ 323: 1033-1034.

Jones, K.E. \& Purvis, A. 1997. An optimum body size for mammals? Comparative evidence from bats. Funct. Ecol. 11: 751-756.

Lee, P.C. 1996. The meanings of weaning: growth, lactation and life history. Evol. Anthro. 5: 87-98.

Leon, D.A., Lithell, H.O., Vågerö, D., Mohsen, R., Berglund, L., Lithell, U-B. \& McKeigue, P.M. 1998. Reduced fetal growth rate and increased risk of death for ischaemic heart disease: cohort study of 15000 Swedish men and women born 191529. BMJ 317: 241-245.

Leutenegger, 1973. Maternal-fetal weight relationships in Primates. Folia Primat 20: 280-293.

Lindenfors, P. 2002. Sexually antagonistic selection on primate size. J. Evol. Biol. 15: 595-607.

Lindström, J. 1999. Early development and fitness in birds and mammals. Trends Ecol. Evol. 14: 343-348.

Luckett, W.P. 1975. Ontogeny of fetal membranes and placenta: their bearing on primate phylogeny. In: Phylogeny of the Primates (W. P. Luckett \& F. S. Szalay, eds), pp. 157-182. Plenum Press, New York.

Lummaa, V. 2003. Early developmental conditions and reproductive success in humans: Downstream effects of prenatal famine, birthweight, and timing of birth. Am. J. Hum. Biol. 15: 370-379.

Møller, A.P. 1991. Sperm competition, sperm depletion, paternal care and relative testis size in birds. Am. Nat. 137: 882-906.

Møller, A.P. \& Briskie, J.V. 1995. Extra-pair paternity, sperm competition and the evolution of testis size in birds. Behav. Ecol. Sociobiol. 36: 357-367.

Macnair, G.A \& Parker, G.A. 1978. Models of parent-offspring conflict. II. Promiscuity. Anim. Behav. 26: 111-122.
Martin, R.D. 1984. Scaling effects and adaptive strategies in mammalian lactation. Symp. Zool. Soc. Lond. 51: 87-117.

Martin, R.D. \& MacLaron, A.M. 1985. Gestation period, neonatal size and maternal investment in placental mammals. Nature 313: 220-223.

Mock, D.W. \& Parker, G.A. 1997. The Evolution of Sibling Rivalry. Oxford University Press, New York.

Parker, G.A. 1970. Sperm competition and its evolutionary consequences in the insects. Biol. Rev. 45: 525-567.

Parker, G.A. 1998. Sperm competition and the evolution of ejaculates: towards a theory base. In: Sperm Competition and Sexual Selection (T. R. Birkhead \& A. P. Møller, eds), pp. 3-54. Academic Press, Toronto, Ontario.

Parker, G.A. \& Macnair, G.A. 1979. Models of parent-offspring conflict. IV. Intra-brood conflict. Anim. Behav. 27: 1210-1235.

Purvis, A. \& Rambault, A. 1995. Comparative analysis by independent contrasts (CAIC): an Apple Macintosh application for analyzing comparative data. Comp. Appl. Biosci. 11: 247-251.

Robbins, C.T. 1993. Wildlife Feeding and Nutrition, 2nd edn. Academic Press, New York.

SAS Institute Inc. 1999. SAS OnlineDoc ${ }^{\circledR}$, version 8. SAS Institute Inc., Cary, NC.

Schülke, O., Kappeler, P.M. \& Zischler, H. 2004. Small testes size despite high extra-pair paternity in the pair-living nocturnal primate Phaner furcifer. Behav. Ecol. Sociobiol. 55: 293-301.

Steenbeek, R. 2000. Infanticide by males and female choice in wild Thomas's langurs. In: Infanticide by Males and Its Consequences (C. P. van Schaik \& C. H. Janson, eds), pp. 153-177. Cambridge University Press, New York.

Trivers, R.L. 1974. Parent-offspring conflict. Am. Zool. 14: 249264.

Vos, R.A. \& Moores, A.Ø. 2005. A dated MRP supertree for the order Primates. Syst. Biol. (in press).

Vrana, P.B., Guan, X., Ingram, R.S. \& Tilghman, S.M. 1998. Genomic imprinting is disrupted in interspecific Peromyscus hybrids. Nat. Gene. 20: 362-365.

Widdowson, E.M. 1974. Immediate and long-term consequences of being large or small at birth: a comparative approach. In: Size at Birth, pp. 65-75. Elsevier, New York.

Received 21 September 2004; revised 30 November 2004; accepted 3 December 2004 